\title{
A Tree-Structured Index Allocation Method with Replication over Multiple Broadcast Channels in Wireless Environments
}

\author{
Sungwon Jung, Member, IEEE, Byungkyu Lee, and Sakti Pramanik
}

\begin{abstract}
Broadcast has often been used to disseminate frequently requested data efficiently to a large volume of mobile units over single or multiple channels. Since mobile units have limited battery power, the minimization of the access and tuning times for the broadcast data is an important problem. There have been many research efforts that focus on minimizing access and tuning times by providing indexes on the broadcast data. In this paper, we have studied an efficient index allocation method for broadcast data with skewed access frequencies over multiple physical channels which cannot be coalesced into a single high bandwidth channel. Previously proposed index allocation techniques have one of two problems. The first problem is that they require equal size for both index and data. The second problem is that their performance degrades when the number of given physical channels is not enough. These two problems will result in an increased average access time for the broadcast data. To cope with these problems, we propose a tree-structured index allocation method. Our method minimizes the average access time by broadcasting the hot data and their indices more frequently than the less hot data and their indexes over the dedicated index and data channels. We present an in-depth experimental and theoretical analysis of our method by comparing it with other similar techniques. Our performance analysis shows that it significantly decreases the average access and tuning times for the broadcast data over existing methods.
\end{abstract}

Index Terms-Mobile databases, multiple broadcast channels, alphabetic Huffman trees, index allocation method, data dissemination, broadcast data.

\section{INTRODUCTION}

$\mathrm{T}$ HE rapid development of wireless network and powerful portable computer technologies has accelerated the development of mobile computing technologies, thus resulting in the increased and widespread use of mobile computing devices. These mobile units will often be disconnected for a prolonged period of time to save the battery power as well as communication cost in the mobile wireless computing environment. In a wireless environment, organizing massive amounts of data on wireless communication networks to provide fast and low-power access to users equipped with palmtops is a new challenge to the data management and telecommunication communities [3], [8].

To cope with this problem, broadcasting has been suggested as a possible solution [1], [6], [11], [12], [15]. Acharya et al. proposed a "Broadcast Disks" technique for structuring the broadcast in a way that provides improved performance for data with a nonuniform access pattern [1]. The basic idea of broadcast disks is to broadcast data items that are most likely to be of interest to a larger section of the client community more frequently than others. However,

- S. Jung is with the Department of Computer Science, Sogang University, Shinsoo-Dong, Mapo-Gu, Seoul, Korea, 121-742.

E-mail: jungsung@ccs.sogang.ac.kr.

- B. Lee is with the Mobile Communication Division, Telecommunication Network Business, Samsung Electronics CO., LTD, 94-1, Imsoo-Dong, Gumi-City, Gyeong-Buk, Korea, 730-350. E-mail: bk75.lee@samsung.com.

- S. Pramanik is with the Dept. of Computer Science and Engineering, Michigan State University, East Lansing, MI 48824-1226.

E-mail: pramanik@cse.msu.edu.

Manuscript received 12 Feb. 2004; revised 5 Aug. 2004; accepted 20 Sept. 2004; published online 17 Feb. 2005.

For information on obtaining reprints of this article, please send e-mail to: tkde@computer.org, and reference IEEECS Log Number TKDE-0045-0204.
Acharya et al.'s method is not likely to work well for semantically related data. Broadcast schemes based on the semantic relationships of data in a single broadcast channel are also proposed [6], [12], [15]. Moreover, the issues of allocating semantically dependent data on multiple broadcast channels are discussed in [7], [11].

There have been many research efforts reported in the literature that focus on improving broadcast methods by providing indexes [9], [14], [16], [17], [22]. Indexes will allow mobile clients to slip into the doze mode most of the time and come into the active mode only when data of interest arrives on the broadcast channel. Most work on improving data broadcast methods aims at enhancing the quality of service based on two performance metrics: tuning time and access time. Tuning time is the amount of time spent by a client in actively listening to the broadcast channel and this has a direct implication on battery power consumption. Access time is the amount of time spent to acquire all the data needed by a client. Imielinski et al. propose a tree-structured index allocation technique on the broadcast data over a single wireless channel, where each data item is considered to have the same access frequency [9]. They showed that providing index-based organization and access to data transmitted over wireless channels can result in significant savings of the battery power consumption. Tan and Yu proposed an index technique for broadcast data with different access frequencies [22]. Their technique is a variation of the work done in [9].

Prabhakara et al. studied efficient ways of broadcasting data to mobile users over multiple physical channels which cannot be coalesced into a lesser number of high-bandwidth physical channels [20]. In their scheme, they use flatstructured indexes which have access and tuning times larger than the tree-structured indexes. Two different 
approaches have been studied on tree-structured indexing techniques for broadcast data over multichannel environments [5], [16], [17], [24]. One approach is to use multi channels such that data and index nodes are interleaved over each channel [5], [16], [24]. The other approach is to partition the multichannels into dedicated data and index channels so that data (index) channels carry only data (index) nodes [17].

In [5], [24], the data and index nodes are allocated in different time slots. Their approaches require using balanced index trees (e.g., B+ tree) whose structures do not capture the relative access frequencies of data nodes. As a result, the tuning and access times for the frequently accessed data become large. In [16], Lo and Chen proposed a scheme that mixes the data and index nodes in the same time slot. They use an unbalanced index tree (e.g., Alphabetic Huffman Trees) to capture the relative access frequencies of data nodes. However, their approach requires equal size of index and data nodes. Since the size of a data node is usually larger (i.e., 10-50 times) than that of an index node, the index node size becomes unnecessarily large in this approach. This will, in turn, dramatically increase the tuning and access times for the broadcast data. Unlike the approaches proposed in [5], [16], [24], Shivakumar and Venkatasubramanian proposed an unbalanced index tree allocation method that partitions the multichannels into dedicated data and index channels [17]. Due to the separation of the index and data channels, this approach does not have the same serious problem as above. However, this approach is not efficient when the number of physical index channels is less than the depth of the index tree.

In this paper, we propose a new tree-structured index allocation method (i.e., a new unbalanced index tree allocation method) for the broadcast data with different access frequencies over multichannels. Our method minimizes the average access time by broadcasting hot data and their indexes more frequently than the less hot data and their indexes. This is not possible in the methods proposed in [16], [17]. Furthermore, our method does not have the above problems due to the following two reasons: First, we adopt the approach proposed in [17] to avoid the equal size requirement of index and data nodes. Second, our method does not require the number of index channels to be equal to the depth of the index tree as in [16]. Note that we do not provide new types of tree-structured indexes, but rather a new tree-structured index allocation method to reduce access time.

The rest of the paper is organized as follows: In Section 2, we discuss a data broadcast method over multiple data channels. We then propose a new tree-structured index allocation method for the broadcast data with skewed access frequencies over index channels in Section 3. In this section, we also discuss how we can access data nodes on data channels from index nodes on index channels. Performance analysis of our method is given in Section 4. In Section 5, we discuss how to partition the given multiple broadcast channels into the optimal number of data and index channels. Finally, Section 6 provides concluding remarks.

\section{Data Broadcast Method over Data Channels}

We propose a new tree-structured index allocation method that partitions the multiple wireless channels into index and data channels. The index and data channels in our method are dedicated to broadcast index nodes and data nodes, respectively. In this section, we first discuss the algorithm to broadcast data nodes over the multiple data channels. A few researches have been done on how to allocate the data over multiple data channels [19], [20], [23]. They assumed the channels in consideration were all symmetric, physical channels with the same bandwidth and the data items were of the same size. They tried to minimize the average access time given multiple broadcast channels by optimally partitioning data among them. Our proposed index allocation scheme is orthogonal to the data allocation method suggested in [19], [20], [23]. In other words, we can use any suggested data allocation method.

In this paper, we proposed a greedy algorithm similar to the one suggested in [20] for simplicity. We also adopt their assumptions. Note that the assumption that data items are of the same size does not make our proposed scheme less flexible. This is because we can always make the equisized data item of different sizes of data items by either partitioning or merging them. Our greedy algorithm is shown in Fig. 1.

It assumes that each data has its own temperature. Data with hot (cold) temperatures implies that they have high (low) access frequencies. Without loss of generality, we assume that data channel $i$ accommodates hotter data than data channel $i+1$ does. ${ }^{1}$ The algorithm in Fig. 1 consists of two steps. The first step sorts the data nodes to broadcast in descending order of their temperatures. In the second step, we compute the average channel temperature by dividing the sum of the temperatures of all data nodes by the number of data channels. It decides how many data nodes can be allocated to data channel 1 . We allocate the first $h$ hottest data nodes to data channel 1 such that the sum of their temperatures either minimally exceeds or is equal to the average data channel temperature. This process continues from channel 2 to $k$. Thus, the broadcast cycle for the data over data channel $i$ is shorter than that over data channel $i+1$. This feature will allow the hotter data nodes to be broadcast more frequently than the colder data nodes, which will reduce the access time for the broadcast data with high access frequencies. Note that the data allocated to the same channel will be considered to have the same broadcast frequencies in this method.

An example of the algorithm is illustrated in Fig. 2, where the number of data channels is 4 . It has 14 data sorted in descending order of their temperatures (i.e., access frequencies) as

$$
E, N, A, D, K, L, M, B, C, I, J, F, G, H,
$$

where temperature $(E)$ and temperature $(H)$ are the hottest and the coldest, respectively. In Step 2, we compute the average data channel temperature by dividing the sum of the temperatures of all 14 data by 4 . Let's assume the average data channel temperature computed is 70 . Then we allocate $\mathrm{E}$ and $\mathrm{N}$ to data channel 1 if we assume the sum of the temperatures of $\mathrm{E}$ and $\mathrm{N}$ either minimally exceed, or is equal to 70. [A, D, K], [L, M, B, C], and [I, J, F, G, H] are, respectively, allocated data channels 2, 3, and 4 in a similar fashion. Note that the broadcast cycle of $[\mathrm{E}, \mathrm{N}]$ over channel 1 is shorter than those over data channels 2,3 , and 4 .

In the next section, we discuss the index allocation method that reflects the data broadcast schedule generated

1. Note that data channel $i$ is named a higher channel than data channel $i+1$. 


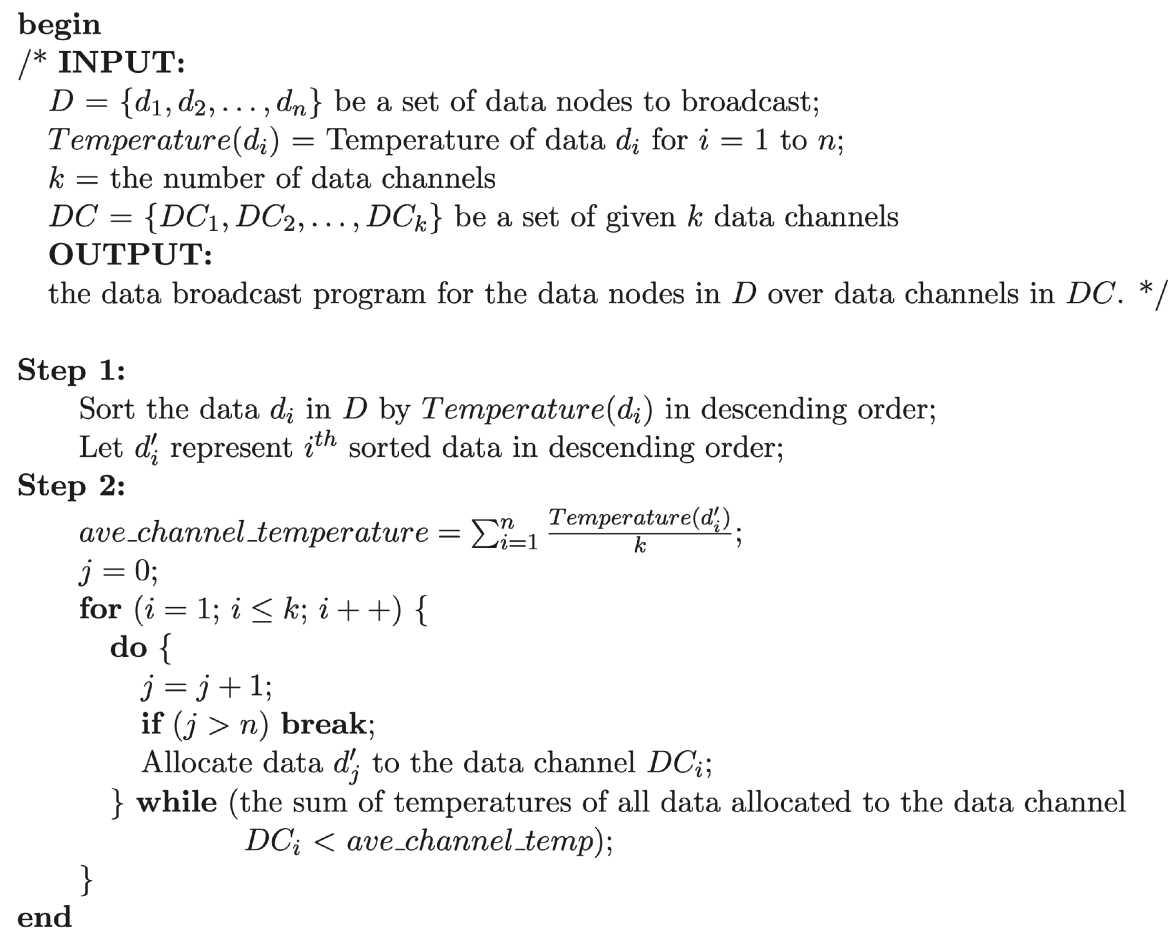

Fig. 1. Data broadcast algorithm over data channels.

by the algorithm in Fig. 1. To minimize the average access time for data with skewed access patterns, our index allocation method requires that the broadcast frequencies of the indexes for data over the data channel $i$ be the same as the broadcast frequencies of the data over the data channel $i$. This requirement guarantees that the indexes for hot data are broadcast more frequently than the indexes for the less hot data. By having this kind of data and index broadcast schedules, the average access time for data over multi channels is minimized.

\section{Index Allocation Method over Index Channels}

In this section, we propose a new tree-structured index allocation method for broadcast data with skewed access frequencies over multichannels. Our method minimizes the average access time by broadcasting hot data and their indexes more frequently than the less hot data and their indexes. Before we discuss our method, we will first discuss the problems of the existing tree-based index allocation methods over multichannels.

\subsection{Existing Tree-Based Index Allocation Methods}

Two different approaches have been studied on treestructured indexing techniques for broadcast data with nonuniform access frequencies over multichannel environments [16], [17]. These techniques use the Alphabetic Huffman tree for indexing the data. The Alphabetic Huffman tree is constructed by the Hu-tucker algorithm [13], [17]. In this algorithm, the data are sorted by their access frequencies and then uses a bottom-up approach to build a tree whose leaf and internal nodes correspond to data and index nodes, respectively. The index nodes are created recursively by combining the first two coldest nodes. ${ }^{2}$ Then, the depth of each data node in the Alphabetic Huffman tree reflects its relative temperature compared to other data nodes. That is, the depth of hot data is low, whereas that of the less hot data is high. This property makes the data at the low depths of the tree have less tuning and access time than those at the high depths of the tree.

An example of an Alphabetic Huffman Tree constructed over the set of data nodes

$$
\{\mathrm{A}, \mathrm{B}, \mathrm{C}, \mathrm{D}, \mathrm{E}, \mathrm{F}, \mathrm{G}, \mathrm{H}, \mathrm{I}, \mathrm{J}, \mathrm{K}, \mathrm{L}, \mathrm{M}, \mathrm{N}\}
$$

of Fig. 2 is shown in Fig. 3. The set of index nodes created by the Hu-tucker algorithm is $\{1,2,3, \ldots, 11,12,13\}$. In the Fig. 3, data nodes $\mathrm{E}$ and $\mathrm{N}$ have the hottest temperatures and data nodes $F$ and $G$ have the coldest temperatures since they are at the highest and lowest depth of the tree, respectively. The Alphabetic Huffman tree in Fig. 3 will be used as a running example throughout the paper.

In [17], Shivakumar and Venkatasubramanian partition the multichannels into dedicated data channels and index channels so that data (index) channels carry only data (index) nodes. They assign one index channel to each level of the Alphabetic Huffman tree. This method has a performance degradation problem when the number of physical index channels is less than the depth of the Alphabetic Huffman tree. In this case, they increase the fanout of the nodes to shorten the depth of the tree or use virtual index channels that are time multiplexed onto the same physical index channels. However, these schemes not only require additional computational overheads, but also

2. The access frequency of an index node is defined as the sum of the access frequencies of its two child nodes. 


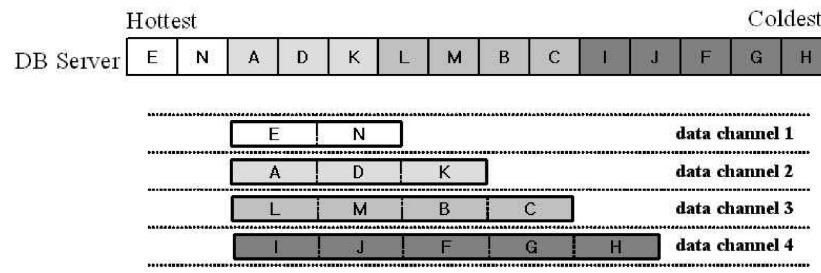

Fig. 2. An example of data broadcast schedule.

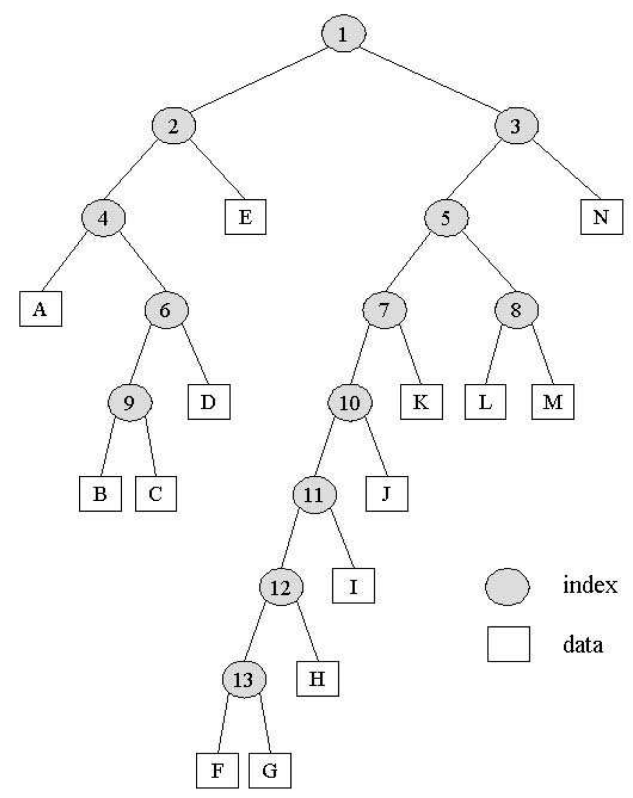

Fig. 3. An example of Alphabetic Huffman tree.

increase the access time for the broadcast data. Fig. 4a shows an example of index allocation method of [17] for the Alphabetic Huffman tree shown in Fig. 3. Since the depth of the tree in Fig. 3 is 8, it has eight index channels, where each channel has index nodes at each level of the tree. It shows an additional problem when the index nodes of the Alphabetic Huffman tree are skewed. This is illustrated in Fig. $4 \mathrm{a}$, where the index nodes 11,12 , and 13 for the cold data $\{\mathrm{F}, \mathrm{G}, \mathrm{H}, \mathrm{I}\}$, for example, are broadcast more frequently than the index nodes 6,7 , and 8 for the hot data $\{\mathrm{D}, \mathrm{K}, \mathrm{L}, \mathrm{M}\}$.

In [16], Lo and Chen use multichannels in such a way that data and index nodes are interleaved over each channel. They create an Alphabetic Huffman tree for the broadcast data first and then create the topological tree adapted for the given multichannels by using the Alphabetic Huffman tree. Last, they assign index and data nodes of the topological tree to the given multichannels. Fig. $4 \mathrm{~b}$ shows an example of the interleaved data and index allocation to six channels by using the topological tree created from the Alphabetic Huffman tree shown in Fig. 3. Their approach has a problem since it requires the index nodes to be of the same size as the data nodes. Note, in practice, that the size of data nodes are usually much larger (i.e., 10-50 times) than that of index nodes [4], [18]. Thus, the index node size becomes unnecessarily large, wasting the bandwidth of channels, which, in turn, dramatically increases the tuning and access times for the broadcast data.

In this paper, we propose a new tree-structured index allocation method for broadcast data with nonuniform access frequencies over multichannels. We minimize the average access time by broadcasting hot data and their corresponding indexes more frequently than the less hot data and their indexes. Our method overcomes the two problems that occurred in [16], [17]. Fig. 4c shows an example of our index allocation method where two index and four data channels are given. Before we discuss our method in detail, we give the assumptions and terminologies that will be used to explain our method in the paper.

\subsection{Assumptions and Terminologies}

In this section, we give the assumptions and the terminologies to be used to explain our method.

- The channels in consideration are assumed to be all symmetric and are physical channels with all having the same bandwidth.

- The time for switching from one channel to another is assumed to be negligible.

- The sizes of data and index nodes need not be equal. However, data nodes are assumed to be all equisized and index nodes are assumed to be all equisized.

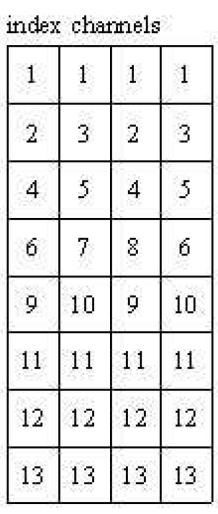

(a)

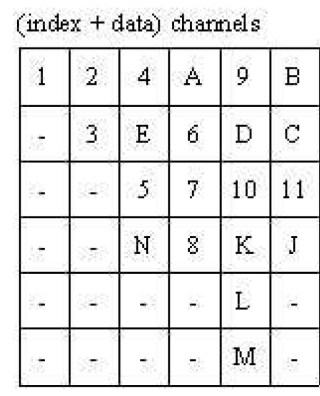

(b) index chantinels

\begin{tabular}{|c|c|c|c|c|c|c|c|c|c|c|c|c|c|c|}
\hline 1 & 2 & $\ldots$ & 13 & 1 & 2 & $\ldots$ & 6 & 1 & 2 & 1 & 2 & $\ldots$ & 13 & $\ldots$ \\
\hline- & 3 & $\ldots$ & - & - & 3 & $\ldots$ & 7 & - & 3 & - & 3 & $\ldots$ & - & $\ldots$ \\
\hline
\end{tabular}

data charmels
\begin{tabular}{|c|c|c|c|c|c|c|c|c|c|c|c|c|c|c|}
\hline E & H & $\ldots$ & E & H & E & $\ldots$ & E & N & E & H & E & $\ldots$ & H & $\ldots$ \\
\hline A & D & $\ldots$ & A & D & K & $\ldots$ & A & S & K & A & D & $\ldots$ & A & $\ldots$ \\
\hline L & M & $\ldots$ & L & M & B & $\ldots$ & B & C & L & M & E & $\ldots$ & M & $\ldots$ \\
\hline J & I & $\ldots$ & H & F & G & $\ldots$ & F & G & J & I & H & $\ldots$ & F & $\ldots$ \\
\hline
\end{tabular}

(c)

Fig. 4. Examples of index allocation methods. (a) Alphabetic Huffman tree-based method. (b) Topological tree-based method. (c) Our index allocation method. 


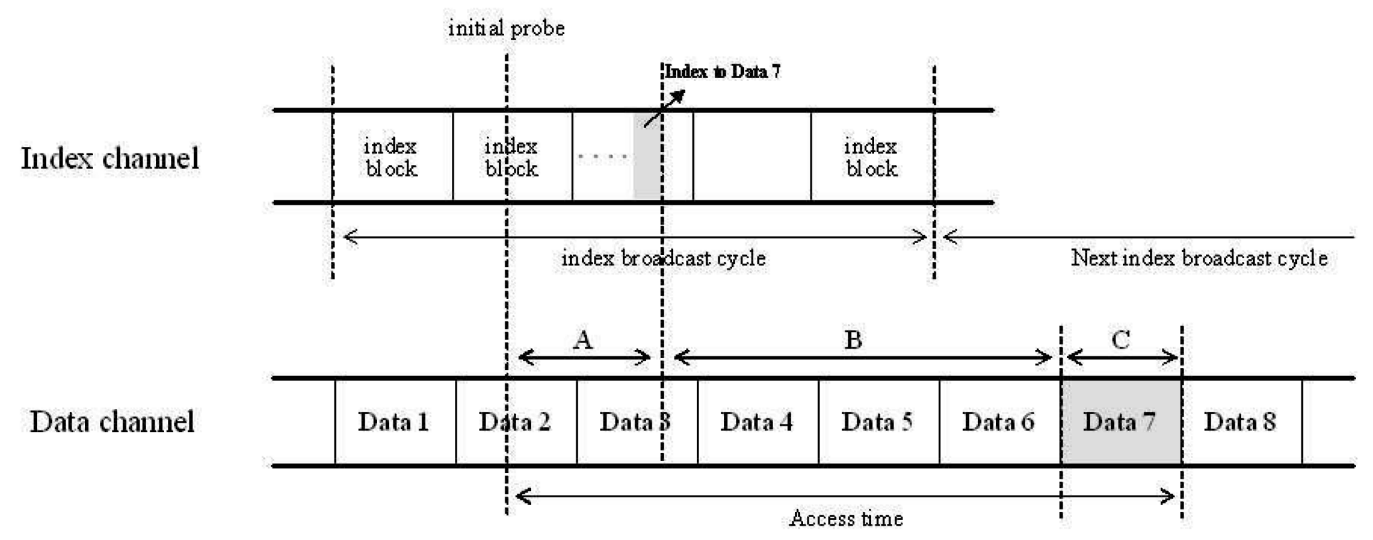

Fig. 5. Examples of access time and index broadcast cycle.

- The mobile clients are assumed to be aware of the way in which they can access the channels. They can only access a single channel at any particular time instance.

- Each index node is assumed to have the pointer data for their child index nodes or data nodes. The pointer data in each index node are represented by the channel number and the time offset pair.

- Access time is the time elapsed from the initial probe (i.e., the time a client request a data) to the point when all required data is downloaded by the mobile client.

- Index broadcast cycle: An index broadcast cycle is the one period of index broadcast.

Fig. 5 shows an example of the access time and the index broadcast cycle. In Fig. 5, a client accesses the index channel to get the index of data 7. It takes $A$ time on this index channel to get the index of data $A$. After that, the client goes into the doze mode for $B$ time until data node 7 is broadcast. Assume that the download time for data 7 is $C$. Then, the access time for data 7 is $A+B+C$.

\subsection{Index Allocation Method}

In this section, we discuss our index allocation method over multichannels. We first introduce a set of basic definitions that will be used in the rest of this paper.

Definition 3.1. Let $D C_{i}$ be the ith data channel. Let $L(v)$ be the level number of a node $v$ in an Alphabetic Huffman Tree. I $L_{1}$ denotes the first ordered index list that contains the sequence of index nodes along the path from the root of an Alphabetic Huffman tree to the data in $D C_{1}$. Then, IL $L_{i}(2 \leq i \leq$ the number of data channels) represents the ith ordered index list which contains the sequence of index nodes along the path from the root of an Alphabetic Huffman tree to the data in $D C_{i}$, excluding all the index nodes in $I L_{j}$ for $j=1, i-1$. Each ordered index list $\left.I L_{i}=<I_{1}, I_{2}, I_{3}, \ldots, I_{q}\right\rangle$ must meet the following two requirements:

1. $L\left(I_{1}\right) \leq L\left(I_{2}\right) \leq L\left(I_{3}\right) \leq \cdots \leq L\left(I_{q}\right)$.

2. When $L\left(I_{j}\right)=L\left(I_{j+1}\right)$, index node $I_{j}$ is on the left of the index node $I_{j+1}$ in the Alphabetic Huffman tree.

Note that we have to create an ordered index list $I L_{i}$ before creating $I L_{i+1}$ due to Definition 3.1. By traversing the Alphabetic Huffman Tree $G(V, E)$ in a topological sorting order ${ }^{3}$ we can easily get all of the ordered index lists. Thus, the time complexity of computing all $I L_{i}$ is $O(|V|+|E|)$, where $|V|$ and $|E|$ represent the total number of nodes and edges in the Alphabetic Huffman tree, respectively.

The indexes in $I L_{i}$ point to the hotter data nodes than those in $I L_{i+1}$ because the data at $D C_{i}$ are hotter than those at $D C_{i+1}$. To incorporate this property into our index allocation method, we also need to broadcast the indexes in $I L_{i}$ over index channels as frequently as the corresponding data in $D C_{i}$ to minimize the access time. To achieve this, it is essential to compute the repetition frequency $R F_{i}$ that $I L_{i}$ have to be broadcast in a single index broadcast cycle. We define the repetition frequency $R F_{i}$ as follows:

Definition 3.2. Let $D X_{i}$ be the number of data allocated in the data channel $D C_{i}$. Let $L C M$ be the Least Common Multiple of $D X_{1}, D X_{2}, \ldots, D X_{N D}$, where $N D$ is the number of data channels. Then, $R F_{i}=\frac{L C M}{D X_{i}}$ represents the repetition frequency that $I L_{i}$ have to be broadcast.

Fig. 6 shows the examples of $I L_{i}$ and $R F_{i}$ obtained from the Alphabetic Huffman tree of Fig. 3. For the data nodes (i.e., $\mathrm{A}, \mathrm{D}, \mathrm{K})$ allocated in the data channel $D C_{2}$, the corresponding ordered index list is $I L_{2}=\langle 4,5,6,7\rangle$ in the figure. This ordered index list meets the two requirements suggested in Definition 3.1 as follows:

1. $(L(4)=3) \leq(L(5)=3) \leq(L(6)=4) \leq(L(7)=4)$.

2. When $L(4)=L(5)$ and $L(6)=L(7)$, the index nodes 4 and 6 are on the left of the index nodes 5 and 7 , respectively.

Note that index nodes 1,2 , and 3 are not included in $I L_{2}$ since they are already in $I L_{1}$. From the figure, we know $D X_{1}=2, D X_{2}=3, D X_{3}=4, D X_{4}=5$, and $L C M=60$. $R F_{2}=20$ means the indices in $I L_{2}$ will be repeatedly broadcast 20 times in a single index broadcast cycle.

As we can see from the above example, the repetition frequencies may result in a broadcast cycle that has a very long period. Therefore, in practice, the repetition frequencies should be chosen with care and, when possible, approximated to simpler ratios. For this, we define the normalized repetition frequencies in the following definition.

Definition 3.3. Let $N D$ be the number of data channels. Given $R F_{1}, R F_{2}, R F_{3}, \ldots, R F_{N D}$, the normalized repetition frequencies are defined as

3. When traversing the Alphabetic Huffman tree, we visit the left child node first before the right child node by the second requirement of Definition 3.1. 
4 Data Channels

\begin{tabular}{|c|c|c|c|c|c|c|c|}
\hline $\mathbf{E}$ & N & & & & $\longrightarrow$ & $\mathbb{I}_{1}:\langle\mathbf{1}, 2,3\rangle$ & $\mathrm{RF}_{1}=60 / 2=30$ \\
\hline A & D & $\mathbf{K}$ & & & & $\mathbb{I}_{2}:\langle 4,5,6,7\rangle$ & $\mathrm{RF}_{2}=60 / 3=20$ \\
\hline L & M & B & C & & & $\mathbb{I}_{3}:\langle 8,9\rangle$ & $\mathrm{RF}_{3}=60 / 4=15$ \\
\hline I & $\mathbf{J}$ & $F$ & G & $\mathbf{H}$ & & $\mathbb{I}_{4}:\langle 10,11,12,13\rangle$ & $\mathrm{RF}_{4}=60 / 5=12$ \\
\hline
\end{tabular}

Fig. 6. Index list and repetition frequency.

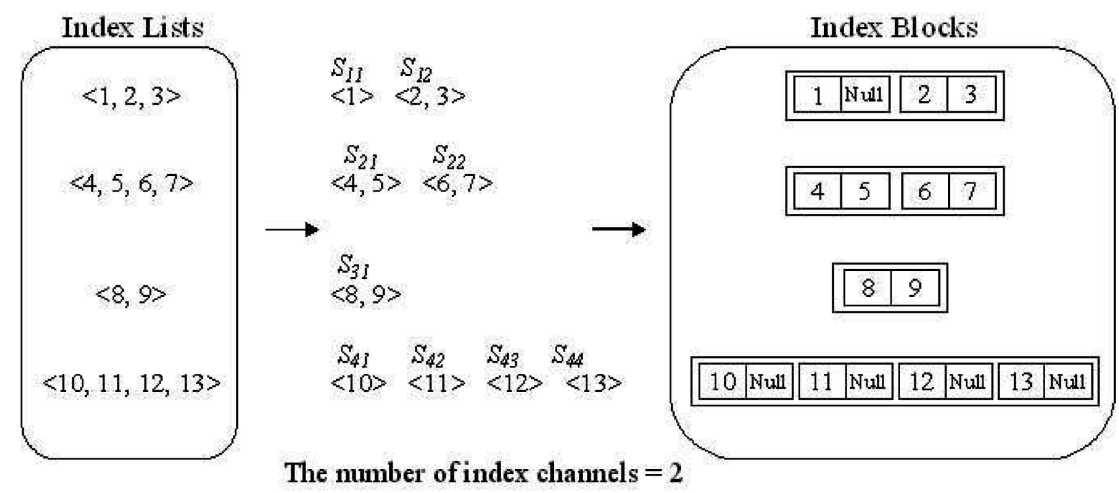

Fig. 7. Examples of index blocks.

$N R F_{1}=\frac{R F_{1}}{R F_{N D}}, N R F_{2}=\frac{R F_{2}}{R F_{N D}}, \ldots, N R F_{N D}=\frac{R F_{N D}}{R F_{N D}}$.

Note that the value of each $N R F_{i}$ is rounded off.

Then, Definition 3.3 gives $N R F_{1}=3, N R F_{2}=2$, $N R F_{3}=1$, and $N R F_{4}=1$ for the above example. Next, we define index block, which will be the basis for our index allocation method.

Definition 3.4. Let $k$ be the number of index channels. A set of consecutive subsequences $S_{i 1}, S_{i 2}, \ldots, S_{i n}$ is defined as the index block $I B_{i}$ for $I L_{i} . S_{i p}$ is named as the pth member of the index block $I B_{i}$, where $1 \leq p \leq n$. The index block $I B_{i}$ must meet the following four requirements:

1. $I L_{i}=S_{i 1}\left\|S_{i 2}\right\| \cdots \| S_{i n}$, where $\|$ represent a concatenation operator.

2. There is no index node $I$ that is in both $S_{i p}$ and $S_{i q}$ for $1 \leq p, q \leq n$ and $p \neq q$.

3. For $p=1, n$, all the index nodes in $S_{i p}$ should not be related to each other in an ancestor-descendant relationship.

4. For $p=1, n$, make $\left|S_{i p}\right|$ be $k$ where $\left|S_{i p}\right|$ represents the number of index nodes in $S_{i p}$. If $\left|S_{i p}\right|<k$, then put a null index at the end of $S_{i p}$ until $\left|S_{i p}\right|$ becomes $k$.

Definition 3.4 uniquely determines the members of index block $I B_{i}$ for $I L_{i}$. This is because all the index nodes in $I L_{i}$ are ordered and they are uniquely partitioned into $S_{i 1}, S_{i 2}, \ldots, S_{i n}$ by the above four requirements. Notice that the time complexity of computing the index block $I B_{i}$ mainly comes from enforcing the third requirement (i.e., avoiding an ancestor-descendant relationship). Let $\left.I L_{i}=\left\langle\cdots, I_{x}, I_{y}, \cdots\right\rangle, S_{i p}=<\cdots, I_{x}\right\rangle$, and $\left|S_{i p}\right|<k$. We can easily check whether $S_{i p}$ can include the node $I_{y}$. That is, we can add the node $I_{y}$ to $S_{i p}$ (i.e., the $p$ th member of the index block $I L_{i}$ ) if the ancestors of node $I_{y}$ is not in
$S_{i p}$. To achieve this, let $d_{i}=L\left(I_{y}\right)-L\left(I_{x}\right)$. Then, we only need to check the following two cases:

- Case 1: When $L\left(I_{x}\right)$ and $L\left(I_{y}\right)$ are the same (i.e., $\left.d_{i}=0\right)$, the index nodes $I_{x}$ and $I_{y}$ do not have an ancestor and descendant relationship. So, $S_{i p}$ becomes $\left.S_{i p}=<\cdots, I_{x}, I_{y}\right\rangle$.

- Case 2: When $L\left(I_{x}\right)$ and $L\left(I_{y}\right)$ are different (i.e., $\left.d_{i}>0\right), S_{i p}$ can include the index node $I_{y}$ if the $d_{i}$ th ancestor node of $I_{y}$ is not in $S_{i p}$.

As we can see, the dominant part of the time complexity of the third requirement comes from Case 2. Let $\left|I L_{i}\right|$ be the size of the $I L_{i}$. Then, the time complexity of computing the index block $I B_{i}$ is $O\left(\left|I L_{i}\right|^{2}\right)$.

Fig. 7 shows an example of the index blocks for the index lists $\langle 1,2,3\rangle,\langle 4,5,6,7\rangle,\langle 8,9\rangle$, and

$$
<10,11,12,13>
$$

obtained from the Alphabetic Huffman tree in Fig. 3 when the number of index channels is 2 . Since we have two index channels, the size of each member of the index block must be 2 and all the elements in each member should not be related to each other by an ancestor-descendent relationship.

To demonstrate how the index block for each index list is obtained, we will show the process of computing the index block $I B_{2}$ for $\left.I L_{4}=<10,11,12,13\right\rangle$. Note that we know the level number of each index node in $I L_{4}$. That is, $L(10)=5, L(11)=6, L(12)=7$, and $L(13)=8$. We first initialize $S_{41}=\langle 10\rangle$. We then go through the remaining index nodes in $I L_{4}$ from left to right by comparing their level numbers. Since $L(11)$ is different from $L(10)$, we compute $d_{4}=L(11)-L(10)=1$. Then, check if the first ancestor node of index node 11 is in $S_{41}=\langle 10\rangle$. Since it is in $\langle 10\rangle$, the index node 11 should not be included in $S_{41}=\langle 10\rangle$. Then, by the fourth requirement of Definition 3.4, we need to insert a Null index at the end of $S_{41}=<10>$. As a result $S_{41}$ 


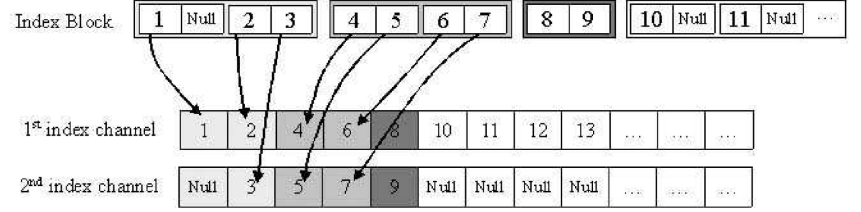

Fig. 8. Allocation of indexes from index blocks to two index channels.

becomes $<10$, Nulll $>$. We can obtain the other members of index block (i.e., $S_{42}=\left\langle 11, N\right.$ ull $>, S_{43}=\langle 12$, Null $>$, $S_{44}=<13$, Null $>$ ) from the rest of the index nodes in $I L_{4}$ in a similar way.

We now discuss our index allocation scheme. Assume that $S_{i p}=\left\langle I_{1}, I_{2}, \ldots, I_{k}\right\rangle$ is a member of the index block for an index list $I L_{i}$ from Definition 3.4. Here, $k$ represents the number of index channels and $I_{1}, I_{2}, \ldots, I_{k}$ represent $k$ indexes. ${ }^{4}$ Since the $k$ indexes are not related to each other by an ancestor and descendant relationship, any part of them do not need to be accessed sequentially for accessing data. This property allows us to broadcast the $k$ indexes to the $k$ index channels simultaneously. That is, we allocate the index $I_{j}$ to the index channel $j$ for $j=1$ to $k$. Fig. 8 shows how we allocate the indexes of the index blocks in Fig. 7 to the two index channels. So far, we have discussed our index allocation method to $k$ multichannels, which is formally described by the algorithm in Fig. 9.

To show how the above algorithm works, we give an example in Fig. 10 where four data channels and two index channels are physically available. We use the data broadcast schedule of Fig. 2 and the Alphabetic Huffman tree of Fig. 3 in this example. It shows the index allocation to two index channels. Since $N R F_{1}=3$, $N R F_{2}=2, N R F_{3}=1$, and $N R F_{4}=1$, the index lists $\left.I L_{1}=\langle 1,2,3\rangle, \quad I L_{2}=\langle 4,5,6,7\rangle, \quad I L_{3}=<8,9\right\rangle$, and $I L_{4}=\langle 10,11,12,13\rangle$ appears 3, 2, 1, and 1 times in the first index broadcast cycle in Fig. 10. In the figure, the horizontal arrows symbolize the pointers to the next index node and the vertical arrows symbolize the pointers to the data node. For example, if a client wants to access data " $H$," it will follow the seven index nodes and one data node such as follows:

$$
1 \rightarrow 3 \rightarrow 5 \rightarrow 7 \rightarrow 10 \rightarrow 11 \rightarrow 12 \rightarrow \mathrm{H} .
$$

We now analyze the time complexity of our index allocation algorithm given in Fig. 9. Since the algorithm consists of four steps, we first compute the time complexity of each step. Step 1 creates index lists by using the Definition 3.1. Its time complexity has been shown to be $O(|V|+|E|)$, where $|V|$ and $|E|$ represent the total number of nodes and edges in the Alphabetic Huffman tree, respectively. The time complexity of Step 2 is $O(N D)$. In Step 3, index blocks are computed by using Definition 3.4 where the time complexity for this computation is shown to be $O\left(\left|I L_{i}\right|^{2}\right)$. Thus, the time complexity of Step 3 is $O\left(\sum_{i=1}^{N D}\left|I L_{i}\right|^{2}\right)$. The time complexity of Step 4 is $O\left(\sum_{i=1}^{N D} N R F_{i} \cdot\left|I B_{i}\right|\right)$. From the analysis, we know that the time complexities of Steps 1 and 3 are dominant. Thus, the time complexity of the algorithm is $O\left(\left(\sum_{i=1}^{N D}\left|I L_{i}\right|^{2}\right)+|V|+|E|\right)$.

4. A sequence of indexes $I_{k}, I_{k-1}, I_{k-2}, \ldots, I_{d}$ may be null indexes by the fourth requirement of Definition 3.4, where $1 \leq d \leq k$.

\subsection{Accessing Data Nodes from Index Nodes}

In this section, we discuss how we can access data nodes on data channels from index nodes on index channels. Each index node stores the pointer data for its child index or data nodes. The pointer data in each index node are represented by $<C N U M, O F F S E T>$ pair, indicating the channel number and the time offset for retrieving its child node. For each index node whose child node is a data node $x$, its corresponding pointer data $<C N U M, O F F S E T>$ also stores the broadcast cycle $B C$ for the data node $x$ over the data channel $C N U M$. That is, $B C$ represents the number of data nodes that must be broadcast over the data channel $C N U M$ before the data node $x$ is broadcast again. To get the next root node of the index tree when a client tunes into the first index channel, each index node also stores the pointer data to the next root node of the index tree appearing in the index channels.

Based on the above discussion, Fig. 11 describes our algorithm that computes the current position of the data node $x$ that is the child node of an index node $y$. Note that we can easily get the index node $y$ by traversing the index tree allocated in the index channels. To demonstrate the above algorithm, we use an example in Fig. 12 where the number of index and data channels is 2 and 4, respectively. Note that the shaded boxes in the index channels represent index nodes pointing to index nodes, whereas the boxes with alphabetic letters in the index channels represent the index nodes pointing to data nodes.

We assume that the size of a data node is 10 times larger than that of an index node. The sizes of index and data nodes are represented in terms of time unit $t$. Thus, the positions of data and index nodes are identified by the time units. In the following figure, we start to broadcast the index and data nodes when $t=0$ over two index and four data channels. Assume that a mobile client wants to access the data node $E$ which is being broadcast over data channel 1 . In order to access data node $E$, the client has to first tune into the first index channel to get the root node of the index tree. Then, it traverses the index tree until it gets the index node pointing to data node $E$. In this example, index node is 2, which is shown as $E$ on index channel 1 in Fig. 12. Let's assume that the index node 2 is being broadcast over the index channel 1 when $t=14$. Note that index node 2 has the information that the data node $E$ is being broadcast over the data channel 1 . Then, we have the input values for the algorithm as follows:

- $x=E, y=2, C N U M=1$, DSize $=10, B C=2$.

- $\quad$ Size_of_Data_Channel $=B C \times D$ Size $=20$;

- $\quad$ current_index_position $=14$ since it is located at $t=14$.

- $O F F S E T=0$.

From the above algorithm, we first have

$$
\text { period_number }=1
$$

and

$$
\text { position }=20 \times 1+0=20 \text {. }
$$

Since current_index_position $=14$ is less than position $=20$, the while loop is not executed and the value of position becomes 20. This means that data node $E$ will be broadcast when $t=20$. After the client gets the location of data node $E$, it goes into the doze mode until the time becomes 20. At $t=20$, it wakes up and gets data node $E$ from the data channel 1 . 


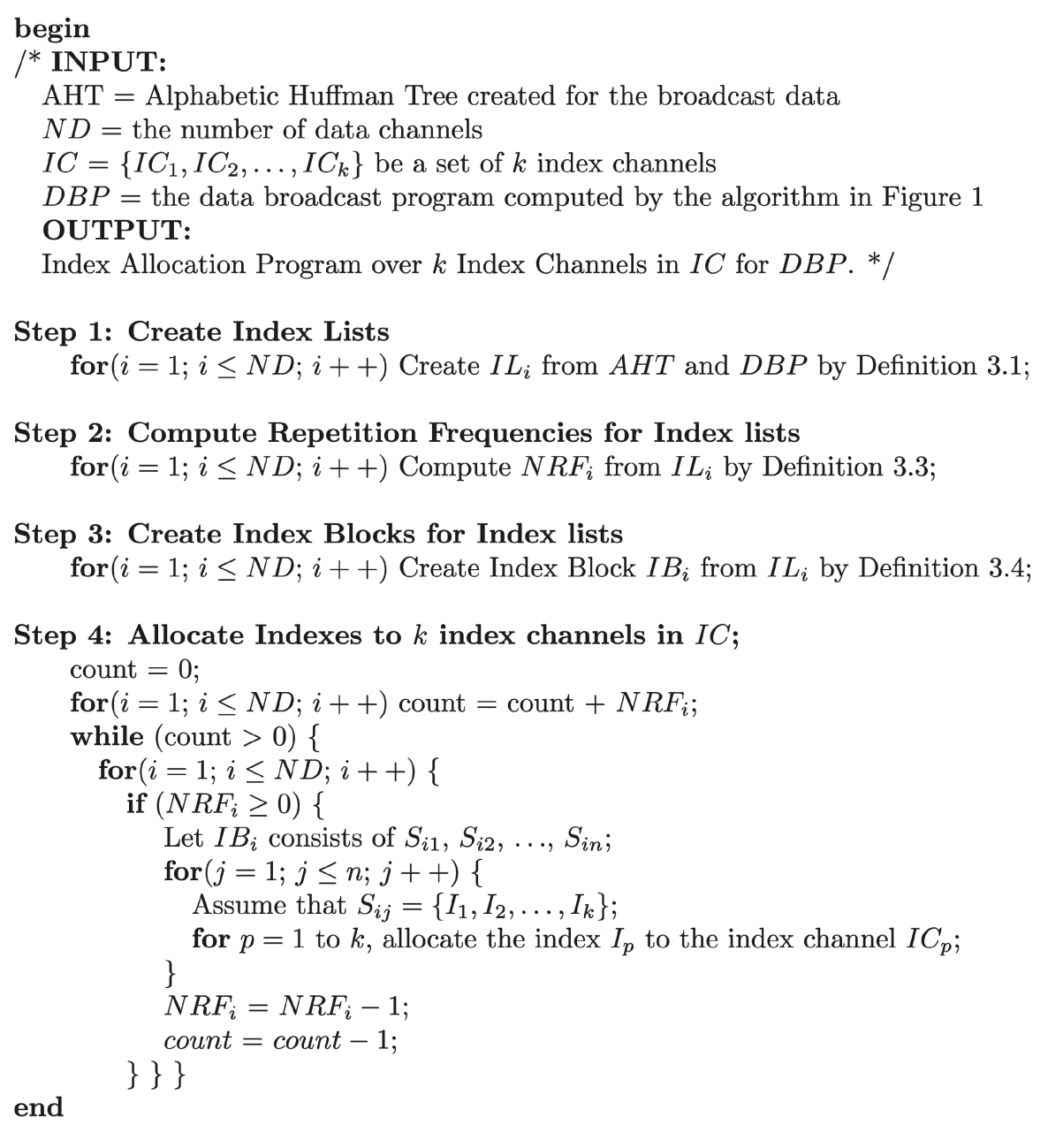

Fig. 9. Index allocation algorithm to $k$ index channels.

\section{Performance Analysis}

We analyze and compare the performance of our method with those of the topological tree-based method [16] and Alphabetic Huffman tree-based method proposed in [17]. For the rest of the paper, we use the abbreviation SIRAH (Separated Index Replication based on Alphabetic Huffman tree) for our method, TOPO (TOPOlogical) for the Topological tree-based method, and ALH (ALphabetic Huffman) for the Alphabetic Huffman tree-based method.

We carried out the simulation for the performance comparison on a Pentium-III 866EB with 512MB memory. The simulator was implemented using CSIM 18 simulation language [2], [21]. To model the nonuniform (or skewed) data access pattern of mobile clients, we use a Zipf distribution with a parameter $\theta$. Note that the Zipf distribution is typically used to model nonuniform access patterns [10], [13], [25]. It produces access patterns that become increasingly skewed as $\theta$ increases. In this simulation, we set the value of $\theta$ to 0.95 to model a skewed access pattern. This access pattern is common in broadcasting environments [10], [13], [25]. The various parameters used in the simulation are tabulated in Table 1.

We chose average access and tuning times of a mobile client as our primary performance metrics. The average access and tuning times are measured by averaging the access and tuning times of a mobile client over 20,000 simulation runs. Note that we measure the access and tuning times in terms of broadcast units. A broadcast unit represents an amount of time taken to broadcast a single index node. For example, it will take 30 broadcast units to broadcast a single data node when $R$ as defined in Table 1 is 30 . In the following section, we analyze the performance of $S I R A H, A L H$, and TOPO over various parameter values.

\subsection{Effects of the Ratio of a Data Node Size to an Index Node Size}

Since the size of an index node is usually very small compared to that of a data node [4], [18], their size differences will affect on the performances of $S I R A H$, $A L H$, and TOPO. Thus, we analyze its effect on the performance of the three methods by varying the ratio of a data node size to an index node size (i.e., $R$ ) from 1 to 50 . Fig. 13 shows that $S I R A H$ performs siginificantly better than $A L H$ and $T O P O{ }^{5}$

In Fig. 13, the average access time of $S I R A H$ increases very slowly as $R$ increases from 1 to 50 . This shows that $S I R A H$ has a very stable performance. In TOPO, as $R$ increases, the bandwidth of channels allocated to index nodes is wasted, thus resulting in a very high average 


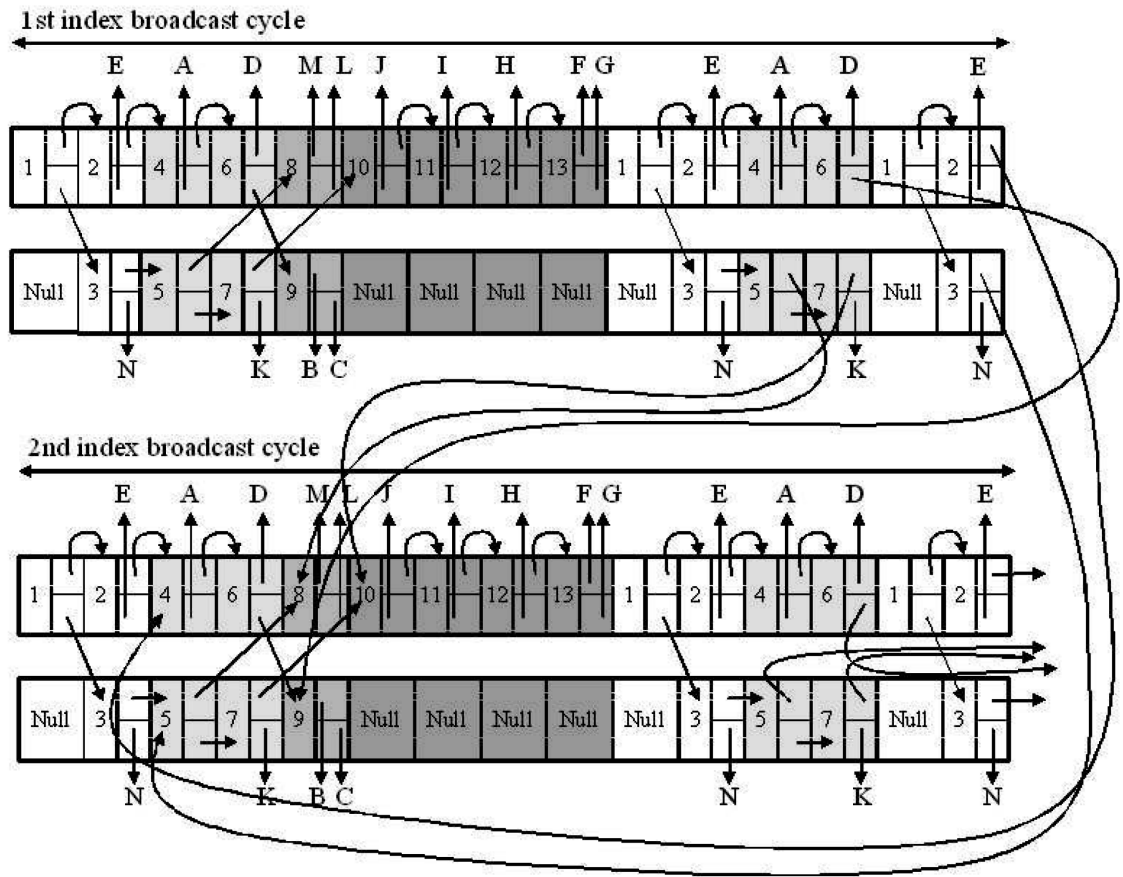

Fig. 10. An example of index allocation generated by the algorithm in Fig. 9.

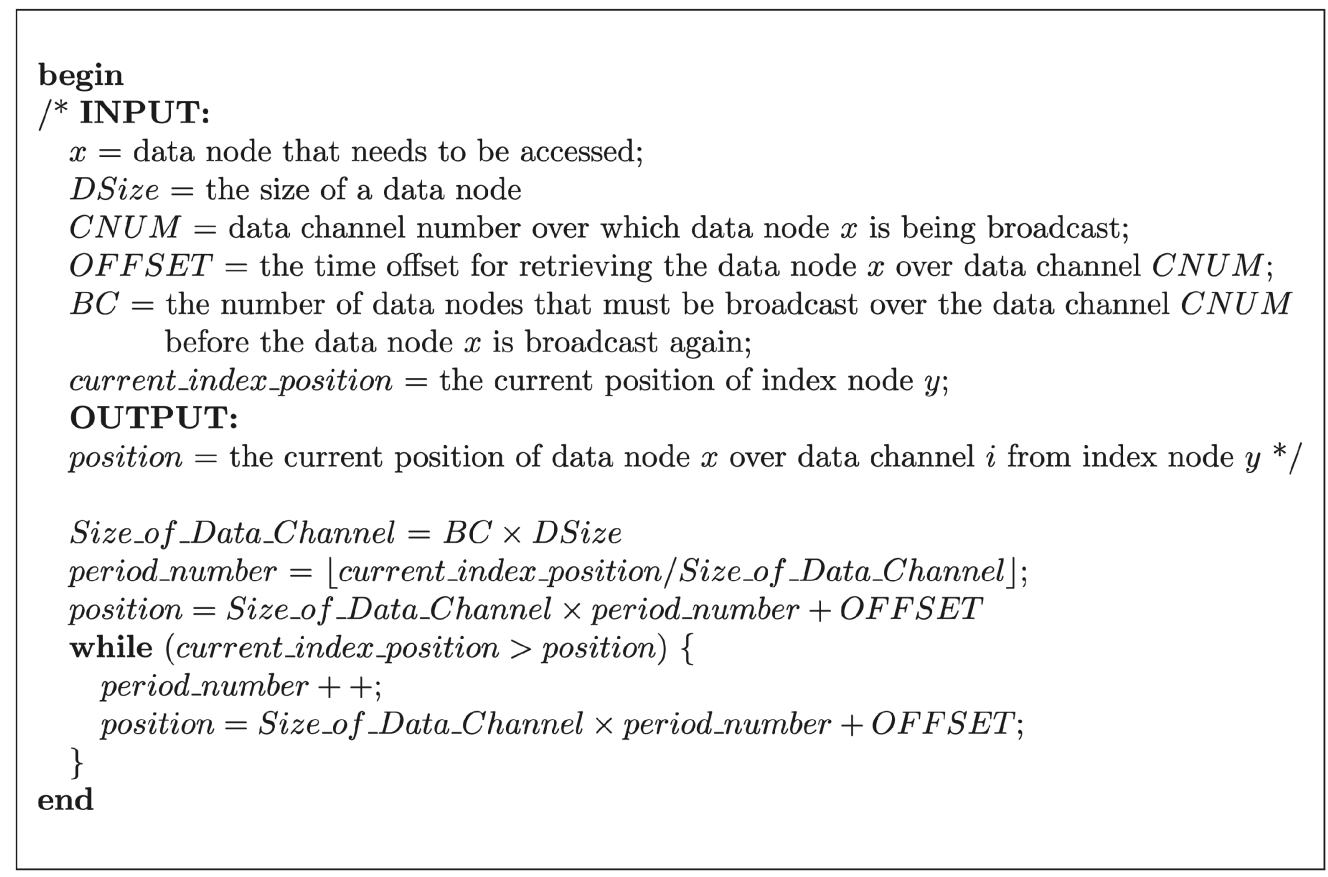

Fig. 11. Accessing a data node from an index node.

access time. However, this problem does not occur in the SIRAH method since we partition the multichannels into index and data channels.

The $A L H$ method also performs better than TOPO because it has dedicated index and data channels like SIRAH. Thus, it clearly shows the advantage of having dedicated index and data channels. However, unlike the $S I R A H$ method, the average access time of $A L H$ is worse

5. When $R=1$, the average access times of $S I R A H$ and TOPO are 853 and 903 , respectively. than that of $T O P O$ when $\mathrm{R}$ is less than 10 . This is because $A L H$ has two inherent performance degradation problems. The first is that it does not broadcast the indexes for hot data more frequently than the indexes for less hot data. The second is that the $A L H$ method does not adapt well when the number of dedicated index channels is less than the depth of the Alphabetic Huffman tree.

Since a good index allocation method should be energyefficient and the tuning time has a direct implication on the battery power consumption, we also analyze the average tuning times of the three methods. As all three of the 


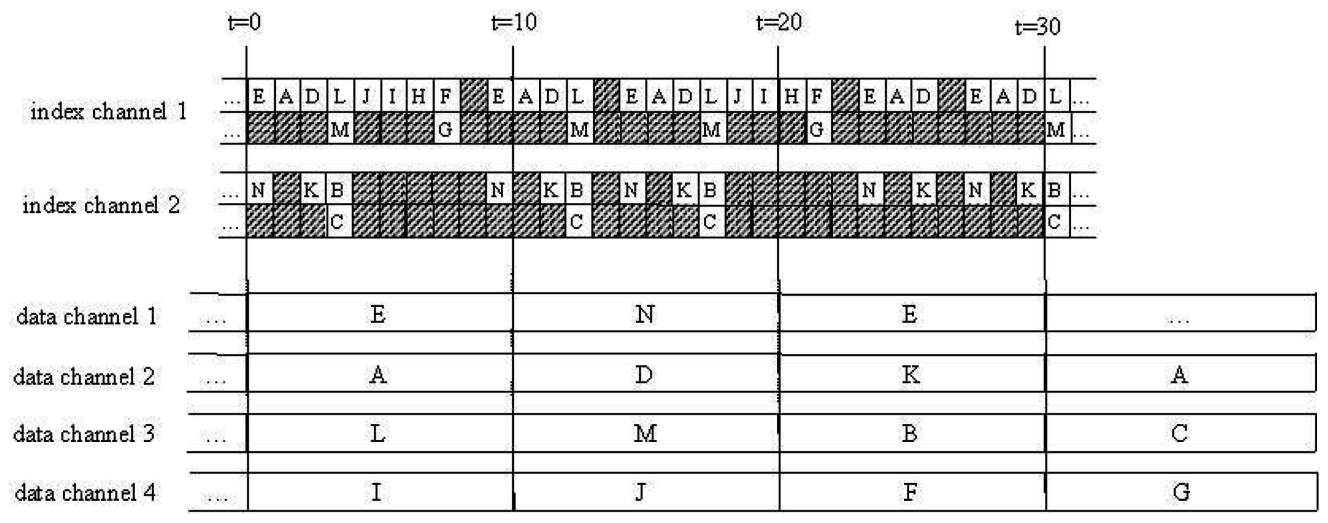

Fig. 12. Mapping index nodes to data nodes over multiple channels.

methods use Alphabetic Huffman tree for their index tree, the total number of traversed index nodes before getting a data node is the same for the three methods. As a result, their average tuning time must be the same if the size of an index node for all three methods is same. Fig. 13 shows that the average tuning times of the $A L H$ and SIRAH methods are the same, while that of the TOPO method is higher. This is because the size of an index node in the TOPO method is not the same as those of the $A L H$ and SIRAH methods, except for when $R$ is 1 . In the TOPO method, an index node size must be increased to as much as the size of a data node. Thus, for example, when $R$ becomes 7 , the index node size in the TOPO method should be increased to 7 times larger than the size of an index node in the SIRAH and $A L H$ methods.

In Fig. 13, the average tuning times of $S I R A H$ and $A L H$ increase very slowly as $R$ increases from 1 to 50 . This shows that $S I R A H$ and $A L H$ have a very stable performance. In $T O P O$, as $R$ increases, the bandwidth of channels allocated to index nodes is wasted, thus resulting in a very high average tuning time. However, this problem does not occur in the SIRAH and $A L H$ methods since the multichannels are partitioned into dedicated index and data channels.

\subsection{Effects of the Number of Broadcast Data}

In this section, we study how increasing the number of broadcast data will affect the performances of the $S I R A H$, $A L H$, and $T O P O$ methods. For the experiment, we fix $R$ to 50 and vary the number of broadcast data from 500 to 3,000. The results are shown in Fig. 14 in terms of the average access time and the average tuning time.

It first shows that the average access time of the $S I R A H$ method is much lower than TOPO and $A L H$ as the number

TABLE 1

Simulation Parameters

\begin{tabular}{||c|c|}
\hline Simulation Parameter & Range \\
\hline \hline the number of data channels & 8 \\
\hline the number of index channels & 2 \\
\hline the total number of channels & 10 \\
\hline the number of broadcast data & 3000 \\
\hline$R=\frac{\text { a data node size }}{\text { an index node size }}$ & $1-50$ \\
\hline$\Theta$ & 0.95 \\
\hline the total number of simulations & 20000 \\
\hline
\end{tabular}

of broadcast data increases. This is because the skewed data access patterns severely deteriorate the performance of $T O P O$ and $A L H$ as the number of broadcast data becomes large. In the $T O P O$ and $A L H$ methods, the broadcast frequencies of hot data and their corresponding index nodes are not high enough to reflect the skewed data access patterns. It becomes even more obvious in the $A L H$ method since it has dedicated index and data channels, like the $S I R A H$ method. As a result, the average access times of the $T O P O$ and $A L H$ methods increase rapidly, as shown in Fig. 14. On the contrary, the SIRAH method overcomes this problem by broadcasting hot data and their corresponding index nodes more frequently than the less hot data and their index nodes in a single broadcast cycle. Thus, the average access time of $S I R A H$ increases very slowly and shows a very stable performance of the SIRAH method. This is shown in Fig. 14.

Fig. 14 also shows the average tuning times of the three methods. Because of the same reasons as explained in Section 4.1, the average tuning times of the $A L H$ and $S I R A H$ methods are the same, while that of the TOPO method is much higher. As the number of broadcast data increases, the height of Alphabetic Huffman Tree also increases, thus resulting in an increased average tuning times of the TOPO, ALH, and SIRAH methods. An interesting thing to note is that the average tuning times of the SIRAH and $A L H$ methods remains almost the same, ${ }^{6}$ while that of $T O P O$ increases noticeably. This phenomenon occurs because a little increase in the height of Alphabetic Huffman Tree tree affects the tuning time of the TOPO method significantly. In other words, the $k$ level increment in the tree height means an $R \times k$ tuning time increment in the TOPO method, whereas, in the $A L H$ and $S I R A H$ methods, it means $k$ tuning time increment.

\subsection{Effects of Varying Access Patterns}

In this section, we analyze how our index allocation scheme SIRAH will behave over other various access patterns. Since user access patterns can vary with time, our index allocation method SIRAH should provide a good stable performance over various user access patterns. Fig. 15 shows the average access and tuning times of the $S I R A H$, $A L H$, and $T O P O$ methods when a Zipf distribution

6. In fact, they are increasing very slowly as the number of data to broadcast increases. Their increase was almost unnoticeable in Fig. 14 due to the plotting of the TOPO method. 

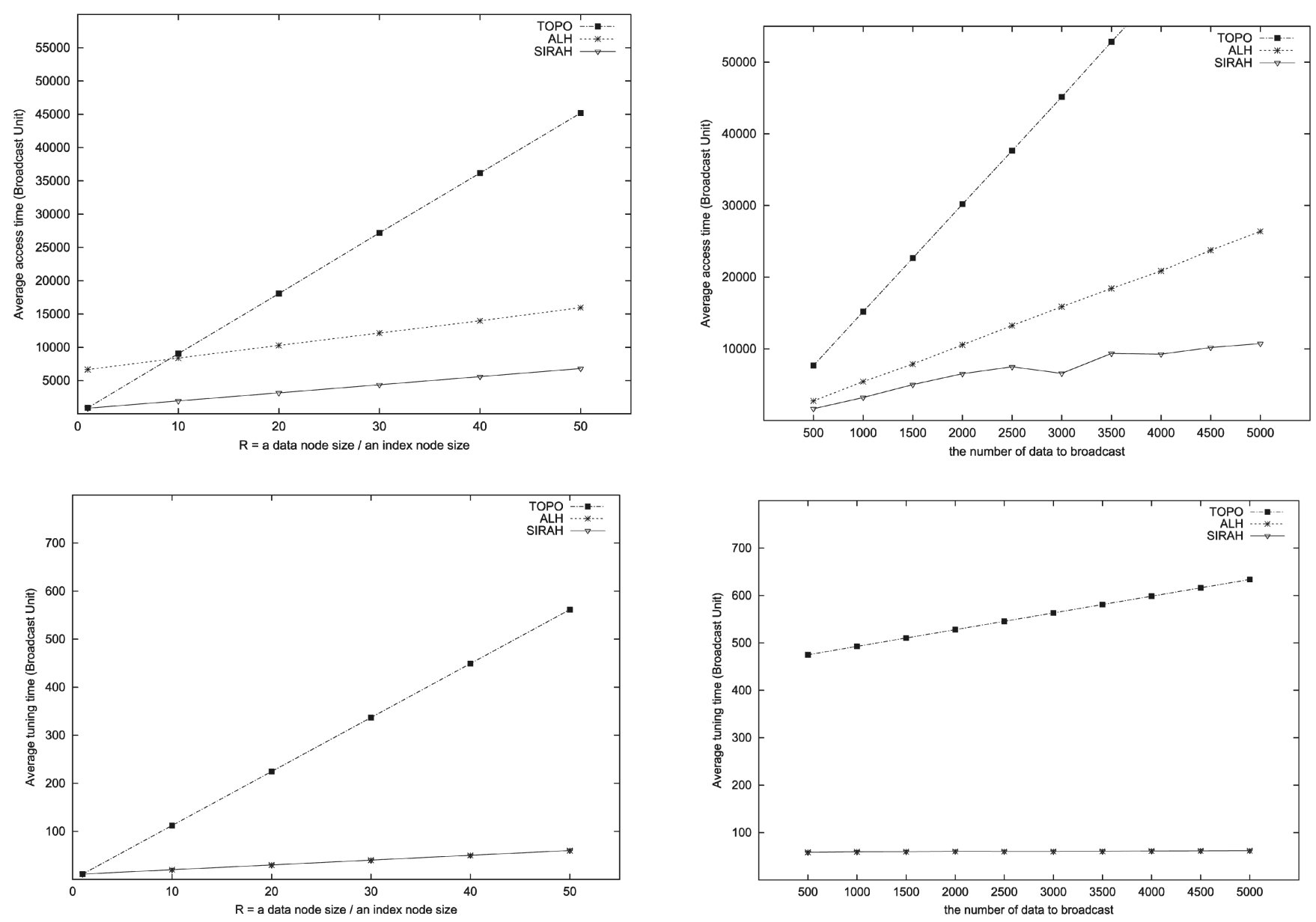

Fig. 13. Performance of TOPO, SIRAH, and $A L H$ when varying $R$.

Fig. 14. Ratio of a data node size to an index node size $=50$.

parameter $\theta$ representing user access patterns is varied from 0.10 to 0.99 .

Fig. 15 shows the average access times of the $S I R A H$, $A L H$, and TOPO methods. The SIRAH method performs better than the other two methods. Unlike the $A L H$ and TOPO methods, its average access time is slowly decreasing as the user access pattern $\theta$ is getting more skewed. However, the average access times of all three methods remains almost unchanged over the various user access patterns. This shows that the Alphabetic Huffman tree they are using for their index trees adapts well to the dynamically varying user access patterns.

We then analyze the average tuning times of the three methods. Similar to the reasons given in Section 4.1, the average tuning times of the $A L H$ and $S I R A H$ methods are also the same, while that of the TOPO method is much higher. The figure also shows how well the average tuning time of the SIRAH method adapt to the dynamically varying user access patterns.

\subsection{Effects of the Number of Data and Index Channels}

So far, we have assumed a given number of index and data channels. In this section, we analyze the performance of the TOPO, ALH, and SIRAH methods in simulations by varying the number of data and index channels. We use the parameters given in Table 2 for the simulations.

Figs. 16 and 17 show the average access and tuning times of a mobile client when the number of channels is $2,3,4,5$, and 10, respectively. For each number of channels, we measure the average access time and tuning time at each possible pair $[a: b]$ of data and index channel partitions. Note that $a$ and $b$ represent the number of data and index channels, respectively. For example, when the number of channels is 4 , our measurements are performed at $[1: 3]$, $[2: 2]$, and $[3: 1]$.

The average access times of the three methods are shown in Figs. 16 and 17. The average access time of the TOPO method is independent of the way data and index channels are partitioned since its scheme does not require dedicated data and index channels. The SIRAH method shows the best average access time when the channels are properly partitioned into data and index channels. For example, we can see from the above two figures that the optimal number of data and index channels for $3,4,5$, and 10 channels is $[2: 1],[3: 1],[4: 1]$, and $[8: 2]$, respectively. From this, we can observe an important property of the $S I R A H$ method. That is, it only requires a small number of index channels to have the optimal performance.

Since an index node size is relatively very small compared to a data node size, the bandwidths of a small number of index channels are large enough to broadcast the entire index tree of the $S I R A H$ method. As a result, the rest of the channels can be dedicated to broadcast data nodes, 

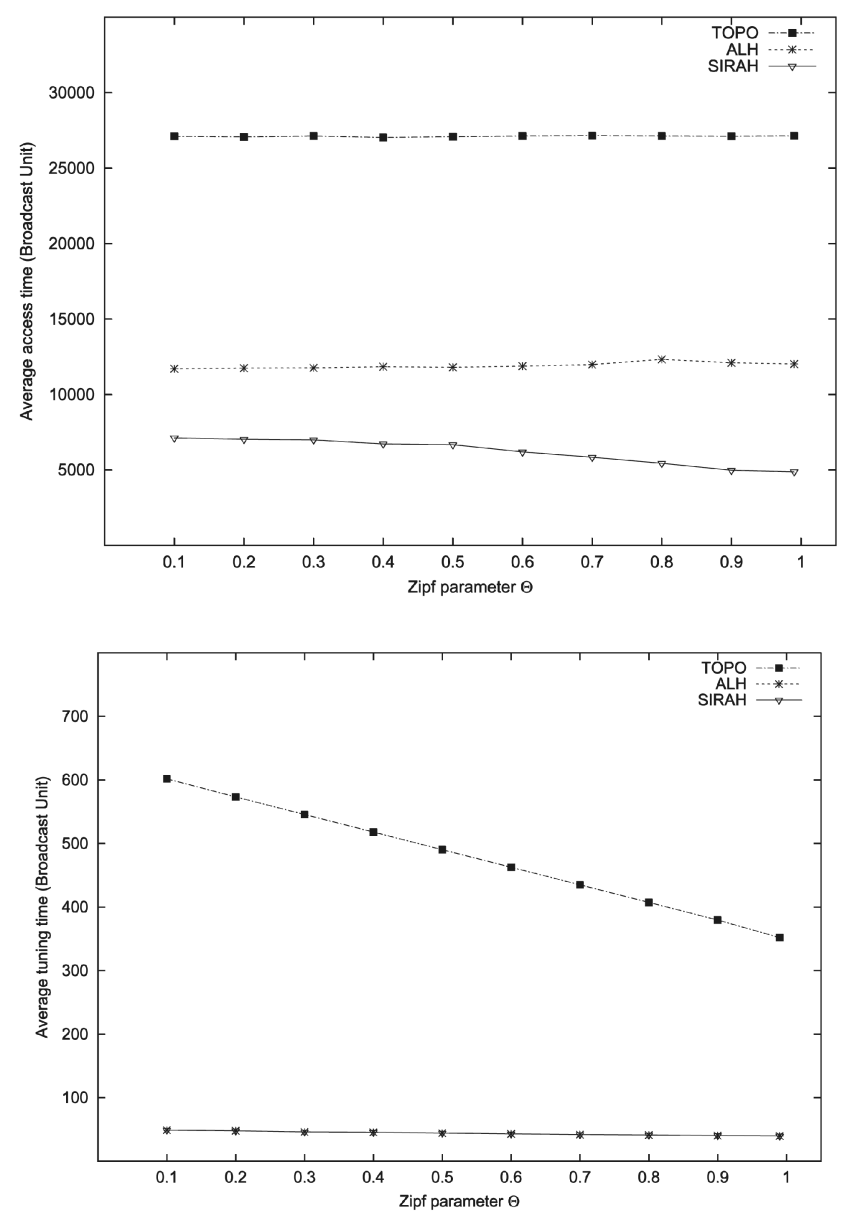

Fig. 15. Varying access patterns.

which will, in turn, reduce the average access times of mobile clients.

Next, we analyze the average tuning times of the three methods. Figs. 16 and 17 show that the average tuning times of the SIRAH and $A L H$ methods are the same and they are better than that of the TOPO method. The figure also shows that all three methods are independent of the way data and index channels are partitioned. Thus, we only need to minimize the average access time of the SIRAH method when we consider the optimal number of index and data channels. Then, the question is how we can determine the optimal data and index channel partition given $n$ channels. In the following section, we present an efficient method to determine the optimal partition.

\section{A Method for Determining the Optimal Number OF DATA AND INDEX ChanNelS}

In this section, we discuss how to partition the given physical broadcast channels into the optimal number of data and index channels. The number of data and index channels have to be determined such that the average access times of mobile clients for the broadcast data should be minimal. We first show an analytical model of the average access time AveAT for broadcast data over the given number of data and index channels. Then, we can determine the optimal partition of data and index channels
TABLE 2

Parameters for Analyzing the Effect of the Number of Data and Index Channels

\begin{tabular}{||c|c|}
\hline Simulation Parameters & Values \\
\hline \hline the total number of channels & $2,3,4,5,10$ \\
\hline the number of broadcast data & 3000 \\
\hline$R=\frac{\text { a data node size }}{\text { an index node size }}$ & 30 \\
\hline & 0.95 \\
\hline the total number of simulation & 20000 \\
\hline
\end{tabular}

by minimizing the average access time AveAT. AveAT consists of the average index access time $I X T$ and the average data access time $D T$. Before defining $I X T$ and $D T$, we list a set of parameters that will be used in this section:

- $c_{d}=$ the number of data channels,

- $c_{i}=$ the number of index channels,

- $c=c_{d}+c_{i}$, where $c$ represents the number of physical channels,

- $n_{j}=$ the number of data to broadcast over data channel $D C_{j}$,

- DSize $=$ the size of a data node,

- $I$ Size $=$ the size of an index node,
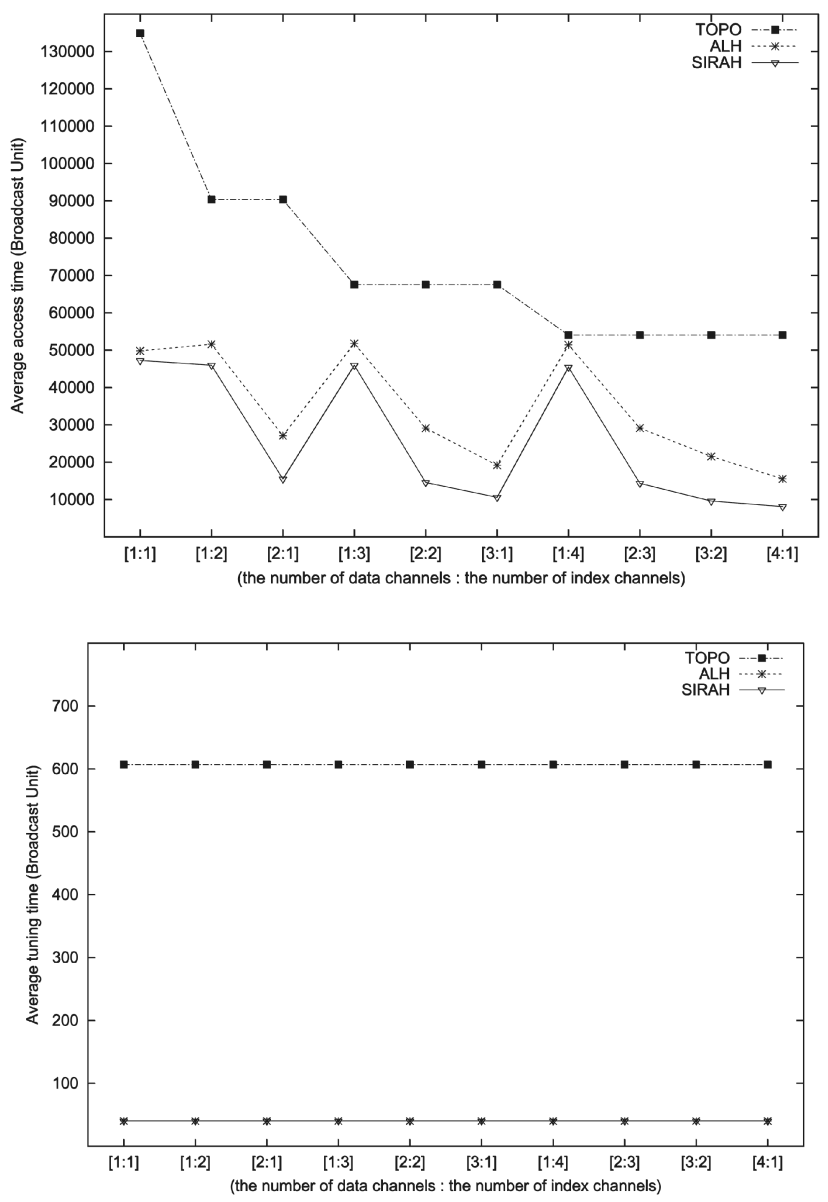

Fig. 16. The number of channels $=2,3,4$, and 5 . 

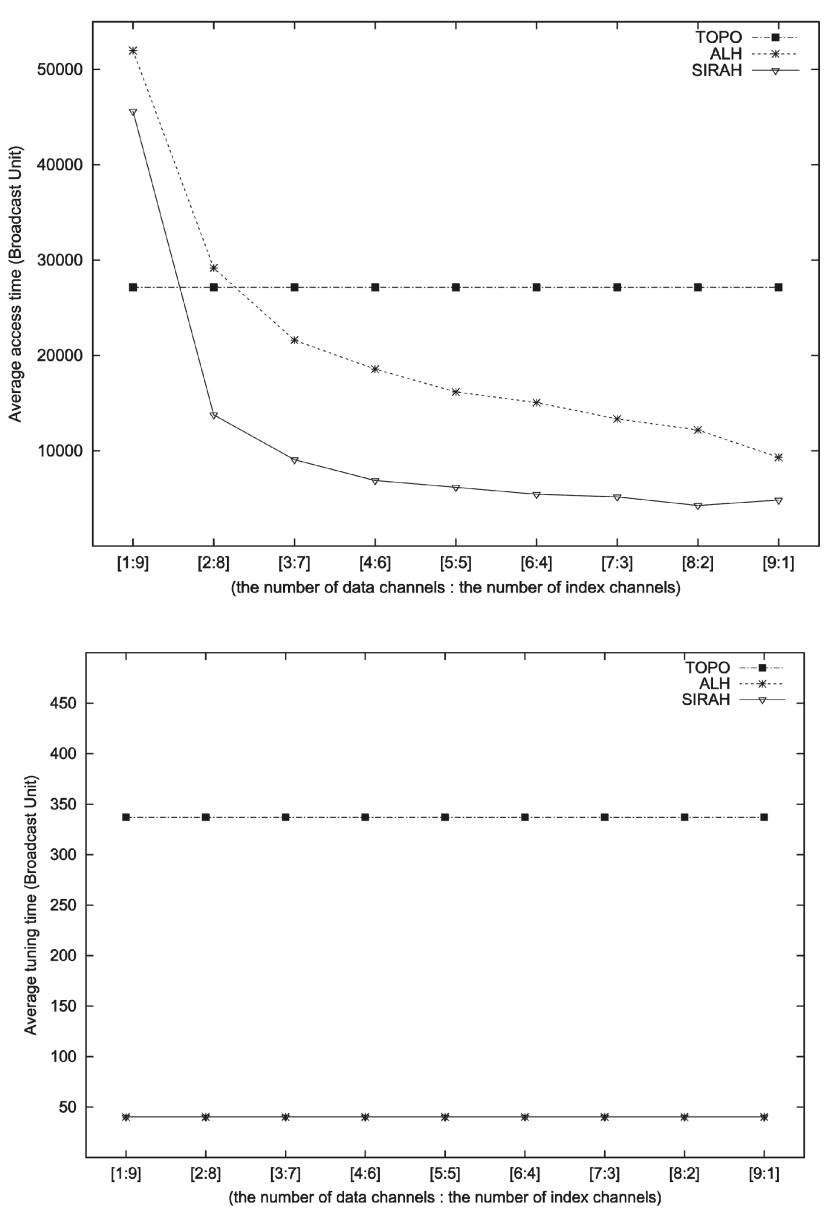

Fig. 17. The number of channels $=10$.

- $\left|I L_{j}\right|=$ the number of index nodes in the index list $I L_{j}$, and

- $\quad N R F=\sum_{j=1}^{c_{d}} N R F_{j}$, where $N R F_{j}$ represents a repetition frequency of $I L_{j}$.

By using the above parameters, Theorem 5.1 formally defines $I X T$ and $D T$.

Theorem 5.1. Let AveAT be the average access time for a broadcast data over $c_{i}$ index and $c_{d}$ data channels. Then, AveAT $=I X T+D T$, where:

$$
\begin{aligned}
& I X T=\frac{I S i z e}{2 \cdot c_{i} \cdot N R F_{1}} \sum_{j=1}^{c_{d}} N R F_{j} \cdot\left|I L_{j}\right|+ \\
& \frac{\text { ISize }}{c_{i}} \sum_{j=1}^{c_{d}}\left[\left\{\sum_{k=1}^{j-1}\left|I L_{k}\right|+\frac{\left|I L_{j}\right|}{2}+\frac{1}{N R F_{j}} .\right.\right. \\
& \left.\left.\sum_{k=1}^{j-1}\left|I L_{k}\right| \cdot\left(N R F_{k}-N R F_{j}\right)\right\} \cdot \frac{N R F_{j}}{N R F}\right] \\
& D T=\frac{D S i z e}{2} \sum_{j=1}^{c_{d}} \frac{N R F_{j}}{N R F} \cdot n_{j} .
\end{aligned}
$$

Proof. In this proof, we ignore the null index nodes and assume the uniform index distribution for the simplicity of the proof. We first prove $I X T$ in Case 1 and then $D T$ in Case 2.
Case 1: $I X T$ consists of $P$ and $L . P$ represents the average time elapsed from the initial probe to get the root of the index tree and $L$ represents the average time taken to get the desired leaf index node after accessing the root index node. To compute $P$, we first need to know the total number of index nodes in a single index broadcast cycle. It is expressed as $\sum_{j=1}^{c_{d}} N R F_{j} \cdot\left|I L_{j}\right|$. Since the number of the root index nodes appears $N R F_{1}$ times in a single index broadcast cycle and all the index nodes are allocated over $c_{i}$ index channels, we can represent $P=\frac{I S i z e}{2 \cdot c_{i} \cdot N R F_{1}} \sum_{j=1}^{c_{d}} N R F_{j} \cdot\left|I L_{j}\right|$. Now, we need to show

$$
\begin{gathered}
L=\frac{\text { ISize }}{c_{i}} \sum_{j=1}^{c_{d}}\left[\left\{\sum_{k=1}^{j-1}\left|I L_{k}\right|+\frac{\left|I L_{j}\right|}{2}+\frac{1}{N R F_{j}} \sum_{k=1}^{j-1}\left|I L_{k}\right|\right.\right. \\
\left.\left.\cdot\left(N R F_{k}-N R F_{j}\right)\right\} \cdot \frac{N R F_{j}}{N R F}\right] .
\end{gathered}
$$

The average time for getting an index node in each index set $I L_{1}, I L_{2}, \ldots I L_{c_{d}}$ is as follows:

- For $I L_{1}: \frac{\left|I L_{1}\right|}{2}$. ISize.

- For $I L_{2}$ :

$$
\begin{aligned}
& \left\{\left|I L_{1}\right|+\frac{\left|I L_{2}\right|}{2}+\frac{1}{N R F_{2}} \cdot\left|I L_{1}\right| \cdot\left(N R F_{1}-N R F_{2}\right)\right\} \\
& \text {. ISize. }
\end{aligned}
$$

- For $I L_{3}$ :

$$
\begin{aligned}
& \left\{\left|I L_{1}\right|+\left|I L_{2}\right|+\frac{\left|I L_{3}\right|}{2}+\frac{1}{N R F_{3}} \cdot\left(\left|I L_{1}\right| \cdot\right.\right. \\
& \left.\left.\left(N R F_{1}-N R F_{3}\right)+\left|I L_{2}\right| \cdot\left(N R F_{2}-N R F_{3}\right)\right)\right\} \\
& \quad \text { ISize. }
\end{aligned}
$$

- $\quad \vdots$ For $I L_{c_{d}}$ :

$$
\begin{aligned}
& \left\{\sum_{k=1}^{c_{d}-1}\left|I L_{k}\right|+\frac{\left|I L_{c_{d}}\right|}{2}+\frac{1}{N R F_{c_{d}}} \sum_{k=1}^{c_{d}-1}\left|I L_{k}\right| .\right. \\
& \left.\left(N R F_{k}-N R F_{c_{d}}\right)\right\} \cdot \text { ISize. }
\end{aligned}
$$

Note that $\left\{\sum_{k=1}^{c_{d}-1}\left|I L_{k}\right|+\frac{\left|I L_{c_{d}}\right|}{2}\right\} \cdot$ ISize represents the average time for getting an index node in the index set $I L_{c_{d}}$ when there are no replicated index nodes. Since our scheme does have the replicated index nodes, the average time for getting an index node in the index set $I L_{c_{d}}$ should be increased, on average, by

$$
\left\{\frac{1}{N R F_{c_{d}}} \sum_{k=1}^{c_{d}-1}\left|I L_{k}\right| \cdot\left(N R F_{k}-N R F_{c_{d}}\right)\right\} \cdot \text { ISize. }
$$

Then, the average time for getting an index node in any of $c_{d}$ index sets over a single index channel is 


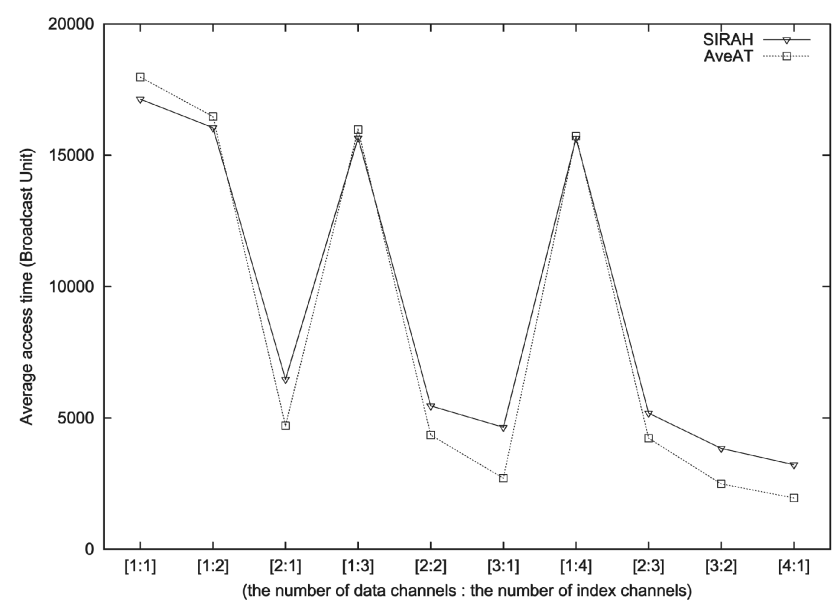

Fig. 18. The number of channels $=2,3,4,5$.

$$
\begin{gathered}
\text { ISize } \cdot \sum_{j=1}^{c_{d}}\left[\left\{\sum_{k=1}^{j-1}\left|I L_{k}\right|+\frac{\left|I L_{j}\right|}{2}+\frac{1}{N R F_{j}} \sum_{k=1}^{j-1}\left|I L_{k}\right|\right.\right. \\
\left.\left.\cdot\left(N R F_{k}-N R F_{j}\right)\right\} \cdot \frac{N R F_{j}}{N R F}\right] .
\end{gathered}
$$

Since we have $c_{i}$ index channels, we need to divide the above expression by $c_{i}$ to get the average time for getting an index node. As a result, we get

$$
\begin{aligned}
L=\frac{I \text { Size }}{c_{i}} \sum_{j=1}^{c_{d}}[ & \left\{\sum_{k=1}^{j-1}\left|I L_{k}\right|+\frac{\left|I L_{j}\right|}{2}+\frac{1}{N R F_{j}} \sum_{k=1}^{j-1}\left|I L_{k}\right|\right. \\
& \left.\left.\cdot\left(N R F_{k}-N R F_{j}\right)\right\} \cdot \frac{N R F_{j}}{N R F}\right] .
\end{aligned}
$$

Based on the above expressions for $P$ and $L$, we show that $I X T=P+L$.

Case 2: We now prove that

$$
D T=\frac{D S i z e}{2} \sum_{j=1}^{c_{d}} \frac{N R F_{j}}{N R F} \cdot n_{j} .
$$

$D T$ represents the average time to get a data node $X$ over $c_{d}$ data channels after getting the corresponding leaf index node over the index channels. It is obvious that the probability of accessing the data channel $D C_{j}$ is $\frac{N R F_{j}}{N R F}$. Since the data node $X$ can be broadcast over one of $c_{d}$ data channels, DT is

$$
\frac{D S i z e}{2} \cdot\left(\frac{N R F_{1}}{N R F} \cdot n_{1}+\frac{N R F_{2}}{N R F} \cdot n_{2}+\cdots+\frac{N R F_{c_{d}}}{N R F} \cdot n_{c_{d}}\right) .
$$

Therefore, $D T=\frac{D \text { Size }}{2} \sum_{j=1}^{c_{d}} \frac{N R F_{j}}{N R F} \cdot n_{j}$.

By the proofs of Cases 1 and 2, it is easy to see that the average access time AveAT for a broadcast data over the $c_{i}$ index and $c_{d}$ data channels is $I X T+D T$.

Given $c$ physical channels, we can determine the optimal number of index and data channels (i.e., $c_{i}$ and $c_{d}$ ) by using the above theorem. That is, we compute a set of AveATs by trying all possible values of $c_{i}$ and $c_{d}$ pairs and then choosing the one giving the minimal value of AveAT. Note that we only need to apply Theorem $5.1(c-1)$ times to compute

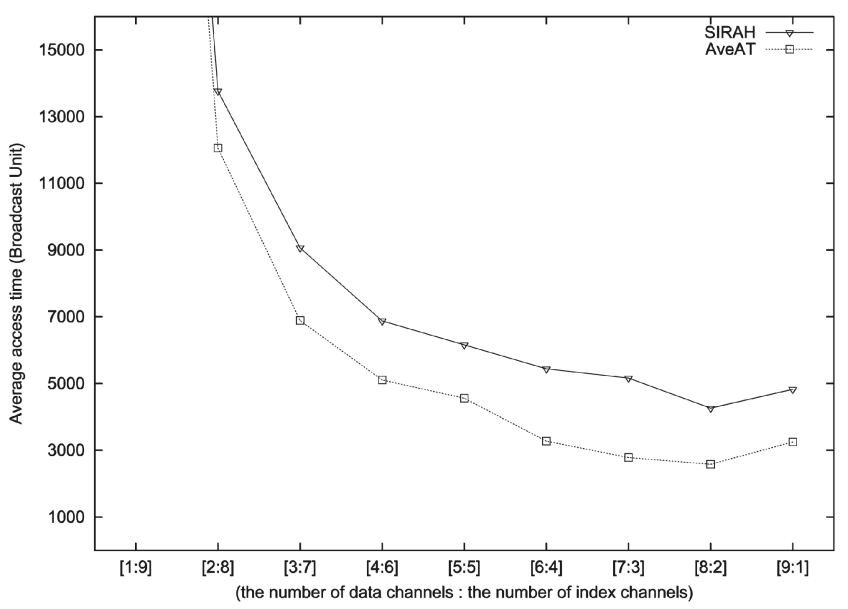

Fig. 19. The number of channels $=10$.

AveATs, although we use enumeration. Since the time complexity of computing AveAT is $O\left(\left(c_{d}\right)^{2}\right)$ and $c_{d}<c$, the time complexity of this approach is $O\left(c^{3}\right)$. In this approach, the whole process of choosing the optimal split has to be reapplied when the data set changes. However, this is not a problem due to the low time complexity (i.e., $O\left(c^{3}\right)$ ).

To verify Theorem 5.1, we first compute AveATs for each possible pair $[a: b]$ of data and index channel partitions by using the same parameters as shown in Table 2. We then plot these AveATs at each pair $[a: b]$ together with the values of the SIRAH method in Figs. 16 and 17. These are shown in Figs. 18 and 19.

They clearly show the effectiveness of Theorem 5.1. For example, when we have 10 channels, Fig. 19 shows that both SIRAH and AveAT have the optimal performance when the numbers of data and index channels are $[8: 2]$. We conjecture that the optimal number of index and data channels will be partially dependent on $R$ (i.e., the ratio of a data node size to an index node size). That is, the smaller $R$ is, the more index channels will be necessary. The reason is that, since a small value of $R$ means a relatively large size of an index node, we need more index channels to broadcast an index tree of the SIRAH method.

\section{Conclusion}

In this paper, we proposed an efficient index allocation method for broadcast data with skewed access patterns over multiple broadcast channels in mobile environments. Our method minimizes average access time by broadcasting hot data and their indexes more frequently than the less hot data and their indexes. Our method further reduces the average access time by not wasting the bandwidth of channels caused by the size difference between an index node and a data node. This is achieved by allocating index and data nodes to dedicated index and data channels, respectively.

We then analyzed the performance of our method over various conditions by comparing it with both an alphabetic Huffman Tree method and a topological tree-based method. The simulation-based results show that our method performs far better than the two methods. We then discussed how to partition the given physical channels into index and data channels to get the optimal performance of the $S I R A H$ 
method. This is based on a newly developed method to efficiently determine the optimal number of index and data channels. We verified the effectiveness of our method through in-depth experimental studies.

\section{ACKNOWLEDGMENTS}

The authors would like to thank the anonymous reviewers for their many insightful comments and suggestions that improved the quality of this paper. They also appreciate the computer simulation programming by Sogang University Computer Science graduate students Sungguen Park, Seunghun Nam, and Seungjung Lee. This research was supported in part by grant No. R01-2003-000-10197-0 from the Basic Research Program of the Korea Science \& Engineering Foundation.

\section{REFEREnCES}

[1] S. Acharya, M. Franklin, S. Zdonik, and R. Alonso, "Broadcast Disks: Data Management for Asymmetric Communication Environment," Proc. ACM SIGMOD Int'l Conf. Management of Data, pp. 199-210, 1995.

[2] Mesquite Software Inc., "CSIM18 Simulation Engine USER'S GUIDE," 2000.

[3] G. Forman and J. Zahorjan, "The Challenges of Mobile Computing," Computer, vol. 27, no. 4, pp. 38-47, Apr. 1994.

[4] H. Leong and A. Si, "Data Broadcasting Strategies Over Multiple Unreliable Wireless Channels," Proc. Fourth Int'l Conf. Information and Knowledge Management, Dec. 1995.

[5] C. Hsu, G. Lee, and A. Chen, "Index and Data Allocation on Multiple Broadcast Channels Considering Data Access Frequencies," Proc. Third Int'l Conf. Mobile Data Management (MDM '02), 2002.

[6] A. Hurson, Y. Chehadeh, and L. Miller, “Object Organization on a Single Broadcast Channel in a Global Information Sharing Environment," Proc. 24th Euromicro Conf., vol. 2, pp. 1021-1028, 1998.

[7] A. Hurson, Y. Chehadeh, and J. Hannan, "Object Organization on Parallel Broadcast Channels in a Global Information Sharing Environment," Proc. IEEE Int'l Performance, Computing, and Comm. Conf. (IPCCC), Feb. 2000.

[8] T. Imielinski and B. Badrinath, "Wireless Mobile Computing : Challenges in Data Management," Comm. ACM, vol 37, no. 10, pp. 18-28, 1994.

[9] T. Imielinski, S. Viswanathan, and B.R. Badrinath, "Data on Air: Organization and Access," IEEE Trans. Knowledge and Data Eng., vol. 9, no. 3, pp. 353-372, May/June 1997.

[10] J. Gray, P. Sundaresan, and S. Englert, K. Baclawski, and P. Weinberger, "Quickly Generating Billion-record Synthetic Databases," Proc. 1994 ACM SIGMOD Int'l Conf. Management of Data, 1994.

[11] J. Juran, A.R. Hurson, N. Vijaykrishman, and S. Boonsiriwattanakul, "Data Organization and Retrieval on Parallel Air Channels, Performance and Energy Issues," Proc. Int'l Conf. High Performance Computing (HiPC 2000), pp. 501-510, 2000.

[12] K. Lee, H. Leong, and A. Si, "Semantic Data Access in an Asymmetric Mobile Environment," Proc. Third Int'l Conf. Mobile Data Management (MDM'02), 2002.

[13] D. Knuth, The Art of Computer Programming, second ed. Addison Wesley, 1998.

[14] W. Lee and D. Lee, "Using Signature Techniques for Information Filtering in Wireless and Mobile Environments," J. Distributed and Parallel Databases, special issue on databases and mobile computing, vol. 4, no. 3, pp. 205-227, July 1996.

[15] K. Lee, H. Leong, and A. Si, "A Semantic Broadcast Scheme for a Mobile Environment Based on Dynamic Chunking," Proc. 20th Int'l Conf. Distributed Computing Systems, pp. 522-529, 2000.

[16] S. Lo and A. Chen, "Optimal Index and Data Allocation in Multiple Broadcast Channels," Proc. 16th Int'l Conf. Data Eng., pp. 293-302, 2000.
[17] N. Shivakumar and S. Venkatasubramanian, "Efficient Indexing for Broadcast Based Wireless Systems," Mobile Networks and Applications, pp. 433-446, 1996.

[18] W. Ni, S. Vrbsky, Q. Fang, and J. Zhang, "Concurrency Control for Mobile Real-Time Databases Using Adaptive Broadcasting," Proc. 20th IASTED Int'l Conf. Applied Informatics, pp. 425-430, Feb. 2000.

[19] W. Peng and M. Chen, "Dynamic Generation of Data Broadcasting Programs for a Broadcast Disk Array in a Mobile Computing Environment," Proc. ACM Conf. Information and Knowledge Management (CIKM), pp. 38-45, 2000.

[20] K. Prabhakara, K. Hua, and J. Oh, "Multi-Level Multi-Channel Air Cache Design for Broadcasting in a Mobile Environment," Proc. 16th Int'l Conf. Data Eng., 2000.

[21] H.D. Schwetman, "CSIM: A C-Based Process Oriented Simulation language," Proc. 1986 Winter Simulation Conf., 1986.

[22] K. Tan and J. Yu, "An Analysis of Selective Tuning Schemes for Nonuniform Broadcast," Data and Knowledge Eng., vol. 22, no. 3, pp. 319-344, 1997.

[23] W. Yee, S. Navathe, E. Omiecinski, and C. Jermaine, "Efficient Data Allocation over Multiple Channels at Broadcast Servers," IEEE Trans. Computers, vol. 51, no. 10, pp. 1231-1236, Oct. 2002.

[24] W. Yee and S. Navathe, "Efficient Data Access to Multi-Channel Broadcast Programs," ACM Conf. Information and Knowledge Management (CIKM '03), pp. 153-160, 2003.

[25] Introduction to Human Ecology. Cambridge, Mass.: Addison Wesley Press, 1949.

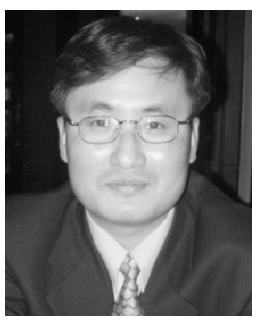

Sungwon Jung received the BS degree in computer science from Sogang University, Seoul, Korea, in 1988. He received the MS and $\mathrm{PhD}$ degrees in computer science from Michigan State University, East Lansing, Michigan, in 1990 and 1995, respectively. He is currently an associate professor in the Computer Science Department at Sogang University. His research interests include mobile databases, mobile computing systems, spatial databases, mobile agent technologies, streaming data processing in ubiquitous computing enviornments, and telematics. He is a member of the IEEE, the IEEE Computer Society, and the ACM.

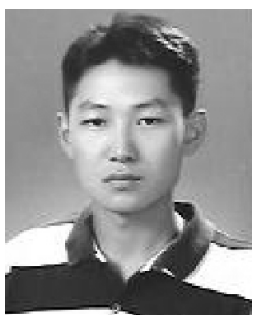

Byungkyu Lee received the BS and MS degrees in computer science from Sogang University, Seoul, Korea, in 2001 and 2003, respectively. He is currently an assistant engineer in the Mobile Communication Division, Telecommunication Network Business, at Samsung Electronics. His research interests include mobile databases and embedded mobile programming.

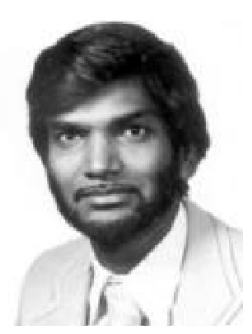

Sakti Pramanik received the BE degree from Calcutta University, the MS degree from the University of Alberta, Edmonton, both in electrical engineering, and the $\mathrm{PhD}$ degree in computer science from Yale University. He is currently a professor in the Department of Computer Science and Engineering at Michigan State University. His research interests include parallel, distributed, and multimedia databases. $\mathrm{He}$ has also done work in bioinformatics.

For more information on this or any other computing topic, please visit our Digital Library at www.computer.org/publications/dlib. 